ORIGINAL ARTICLE

\title{
CagA in Barrett's oesophagus in Colombia, a country with a high prevalence of gastric cancer
}

\author{
M Kudo, O Gutierrez, H M T El-Zimaity, H Cardona, Z Z Nurgalieva, J Wu, D Y Graham
}

J Clin Pathol 2005;58:259-262. doi: 10.1136/jcp.2004.022251

See end of article for authors' affiliations

Correspondence to Dr H M T El-Zimaity, Rm 3A-320 (1 11 lD), Veterans Affairs Medical'Center, 2002 Holcombe Blvd, Houston, Texas 77030 , USA; hzimaity@bcm.tmc. edu

Accepted for publication 28 September 2004

\begin{abstract}
Background: In the USA, atrophic gastritis and gastric cancer are rare, whereas gastro-oesophageal reflux disease (GERD) is common. Infection with Helicobacter pylori, especially a CagA positive strain, is unusual in patients with GERD/Barrett's oesophagus in the USA.

Aim: To examine the relation between Barrett's oesophagus and CagA positive $H$ pylori in Colombia, a country with a high prevalence of $\mathrm{CagA}$ positive $H$ pylori associated atrophic gastritis and gastric cancer. Methods: Helicobacter pylori and CagA status was determined among Colombian patients with long segment Barrett's oesophagus and a control group with simple $H$ pylori gastritis. Helicobacter pylori status was determined using a triple stain and CagA status was determined by immunohistochemistry using a specific rabbit anti-CagA serum.

Results: Gastric and oesophageal mucosal biopsies were obtained from 51 patients -39 men (mean age, 57.8 years; SD, 13.1) and 12 women (mean age, 51.8 years; SD, 14.4)-with documented long segment Barrett's oesophagus. The results were compared with 24 Colombian patients with $H$ pylori gastritis without oesophageal disease. Thirty two patients with Barrett's oesophagus had active $H$ pylori infection. CagA status was evaluated in a subset of $23 \mathrm{H}$ pylori infected patients with Barrett's oesophagus, and was positive in eight of these patients compared with 19 of 24 controls $(p=0.01$ ).

Conclusions: Although most Colombian patients with Barrett's oesophagus had $H$ pylori infection, CagA positive infections were unusual. These data illustrate how consistent corpus inflammation reduces acid secretion, which prevents Barrett's oesophagus among those with abnormal gastro-oesophageal reflux barriers.
\end{abstract}

$\mathrm{B}$ arrett's oesophagus consists of a metaplastic transformation of the lining of the distal oesophagus in which the normal squamous epithelium is replaced by a specialised columnar epithelium with goblet cells. ${ }^{1}$ Barrett's oesophagus is thought to be the precursor lesion to adenocarcinoma of the oesophagus, which has become the most rapidly increasing cancer among white men in Western countries. ${ }^{2-4}$ A search for a possible association between Barrett's oesophagus and Helicobacter pylori infection was logical because $H$ pylori infection causes gastro-duodenal mucosal inflammation, leading to metaplastic transformation of the gastric epithelium and to gastric cancer. ${ }^{56}$ However, when $H$ pylori is present in the oesophagus, it is typically found associated with gastric-type epithelium, and not on specialised metaplastic Barrett's epithelium, and the consensus is that a direct role for $H$ pylori in adenocarcinoma is very unlikely. ${ }^{7-12}$

\footnotetext{
"Helicobacter pylori infection in a patient with an abnormal reflux barrier would depend in part on the pattern and severity of gastritis"
}

Barrett's oesophagus is a complication of gastro-oesophageal reflux disease (GERD). The presence of gastro-oesophageal reflux causing clinical manifestations (disease) is related to the duration and causticity of the refluxate (the oesophageal acid load). ${ }^{13}$ Helicobacter pylori infection directly affects the stomach's ability to produce acid, ${ }^{13}{ }^{14}$ and the recognition that the prevalence of $H$ pylori infection was decreasing in the same population in which oesophageal adenocarcinoma was increasing (white men) suggested that this inverse association may reflect loss of $H$ pylori induced damage to the stomach. ${ }^{15}$ The presence of $H$ pylori infection also results in dysregulation of the normal acid inhibitory reflexes associated with low antral $\mathrm{pH}$, leading to prolonged acid secretion after a meal, such that antral predominant $H$ pylori gastritis is associated with increased acid production. ${ }^{16}$ In contrast, $H$ pylori induced inflammation in the acid secreting gastric corpus impairs the ability of parietal cells to produce acid, and eventually may result in loss of glandular elements and a permanent reduction in acidity. Therefore, $H$ pylori infection in corpus gastritis has been likened to a biological antisecretory agent. ${ }^{14}$

Theoretically, the effect of $H$ pylori infection in a patient with an abnormal reflux barrier would depend in part on the pattern and severity of gastritis. ${ }^{13}$ One would expect that patients without $H$ pylori infection and/or patients with antral predominant gastritis and normal or increased acid secretion would have the highest risk for GERD and Barrett's oesophagus. The risk would decrease progressively with increasing corpus gastritis, and would essentially be absent in patients with atrophic corpus gastritis. ${ }^{13}$ Helicobacter pylori also vary with respect to their ability to cause mucosal inflammation. Helicobacter pylori that contain the cag pathogenicity island (CagA positive) cause more inflammation than those lacking the island, so that with all other things being equal, infection with CagA positive $H$ pylori should be associated with the lowest incidence of clinical GERD and of Barrett's oesophagus. ${ }^{13}{ }^{14}$ The clinical observations are consistent with this reasoning. ${ }^{17-20}$

Both GERD and adenocarcinoma of the oesophagus are rare in countries where atrophic gastritis is common, and this has been attributed to the fact that $H$ pylori associated inflammation and/or destruction of the gastric corpus reduces the oesophageal acid load to below the threshold

Abbreviations: GERD, gastro-oesophageal reflux disease 
that will allow the development of either symptomatic GERD or Barrett's oesophagus. ${ }^{13}$ Nonetheless, Barrett's oesophagus can be found in regions where both CagA positive $H$ pylori infections and gastric cancer are common. Colombia is one such country. ${ }^{21-24}$ This study was designed to test whether the inverse association between $H$ pylori, especially CagA positive $H$ pylori, and Barrett's oesophagus is also present in Colombia. We hypothesised that patients with Barrett's oesophagus in Colombia would be primarily $H$ pylori negative, and because most Colombians with $H$ pylori infection are CagA positive, those with $H$ pylori infection would be infected with a CagA positive strain. To test these hypotheses, we evaluated the relation between Barrett's oesophagus and CagA positive $H$ pylori in Colombia.

\section{METHODS}

\section{Patients}

Our study population consisted of consecutive patients with long segment Barrett's oesophagus (at least $3 \mathrm{~cm}$ above the gastro-oesophageal junction) examined at the gastroenterology outpatient unit of the department of internal medicine of the National University, Bogotá, Colombia. Patients were excluded if they had had $H$ pylori eradication treatment, gastric or gallbladder surgery, or if they did not give written consent to participate in our study. Patients were not excluded if they were receiving antisecretory drugs. The protocol was approved by the ethical committee of the department of internal medicine, National University of Colombia.

\section{Biopsy specimens}

Mucosal biopsy specimens were obtained from the four quadrants of the oesophagus and additional biopsies were obtained from the gastric corpus and antrum. Each specimen was placed in a formalin bottle and routinely processed. For a control group, gastric biopsies were obtained from Colombian patients diagnosed with simple gastritis (for example, no coexisting ulcer disease, oesophagitis, or gastric cancer). Glass slides and/or paraffin wax embedded tissue blocks were shipped to the gastrointestinal mucosal pathology laboratory at Baylor College of Medicine in Houston, Texas, USA.

Serial sections were stained with a triple stain. ${ }^{25-27}$ Each specimen was reviewed by an experienced gastrointestinal pathologist (HEZ) and a trainee (MK) and scored using a visual analogue scale from 0 (absent/normal) to 5 (maximal intensity) for $\mathrm{H} \mathrm{pylori}$, active and chronic inflammation, and intestinal metaplasia and atrophy. ${ }^{28}$

\section{CagA antisera}

Two to three month old specific pathogen free New Zealand white rabbits of either sex (Charles River Laboratories, Wilmington, Massachusetts, USA) received three intramuscular injections of a mixture of equal volumes of $50 \mu \mathrm{g}$ of purified recombinant CagA (Chiron-RIBA, Chiron Corp, Emeryville, California, USA) H pylori protein and Freund's adjuvant in a final volume of $5 \mathrm{ml}$. Freund's complete adjuvant was used for the first immunisation and Freund's incomplete adjuvant was used subsequently.

\section{Immunohistochemistry studies}

For immunophenotyping of CagA antisera status, $4 \mu \mathrm{m}$ thick sections of formalin fixed, paraffin wax embedded tissue were stained using a modified streptavidin-biotin complex method with antigen retrieval. Briefly, slides were pretreated for antigen retrieval with proteinase $\mathrm{K}$ for two minutes, then steamed for 20 minutes in a Black and Decker steamer in $10 \mathrm{mmol} /$ litre citrate buffer ( $\mathrm{pH}$ 6.0), followed by cooling for 30 minutes. The following reagents were then added sequentially (at room temperature unless stated otherwise): inhibitor of endogenous peroxidase; biotin blocking system (Dako, Carpinteria, California, USA); primary antibody, diluted $1 / 50$, overnight at $37^{\circ} \mathrm{C}$; biotinylated goat antirabbit secondary antibody, diluted $1 / 1000$ in 5\% skimmed milk in phosphate buffered saline, for one hour; avidin-horseradish peroxidase complex for 30 minutes; and 3,3'-diaminobenzidine tetrahydrochloride for five minutes. Slides were counterstained with Alcian blue. Sensitivity and specificity were tested using gastric biopsies from patients in whom the CagA status was unequivocally known based on polymerase chain reaction on culture grown colonies.

\section{Statistical methods}

Analyses used SigmaStat 3.0 (SPSS, Chicago, Illinois, USA). Fisher's exact test or, when appropriate, the $\chi^{2}$ test (both two tailed), were used for comparison of proportions. Significance of differences and associations was determined by $\mathrm{p}$ values of less than 0.05 .

\section{RESULTS}

Gastric and oesophageal mucosal biopsies were obtained from 51 patients (39 men: mean age, 57.8 years; SD, 13; 12 women: mean age, 51.8 years; SD, 14) with long segment Barrett's oesophagus. One patient had high grade dysplasia, and two had low grade dysplasia in Barrett's epithelium. In addition to Barrett's oesophagus, four other patients had duodenal ulcers. The control group consisted of 24 Colombian patients with $H$ pylori gastritis.

\section{CagA immunostaining}

Gastric mucosal biopsy specimens from 36 patients with active $H$ pylori infection in whom the infecting $H$ pylori had previously been typed in relation to CagA status using the polymerase chain reaction (24 Colombian patients, with 19 being CagA positive and five CagA negative; 12 US patients, comprising six with CagA positive and six with CagA negative infections) were tested. In US cases there were no false positives or false negatives (sensitivity and specificity, $100 \%)$. In the Colombian cases, there were two false positives (sensitivity and specificity were $100 \%$ and $60 \%$, respectively), possibly related to mixed infections. ${ }^{29}$ Overall, the immunostaining had a sensitivity of $100 \%$ and a specificity of $81.8 \%$, such that the proportion with CagA positive infections might be overestimated.

\section{Helicobacter pylori and CagA status among patients with Barrett's oesophagus}

Glass slides were obtained from 51 patients with Barrett's oesophagus. Paraffin wax embedded tissue blocks were available from 38 patients; only one block was received for 15 patients. Examination of the gastric mucosal biopsy specimens from the 51 patients with long segment Barrett's showed that 32 had active $H$ pylori infection. CagA status was evaluated in a subset of 23 patients for whom a tissue block was available from both the antrum and corpus, and in whom the triple stain confirmed the presence of active $H$ pylori infection; eight were infected with CagA positive $H$ pylori. In the control $H$ pylori infected patients, 19 of 24 were infected with CagA positive $H$ pylori $(\mathrm{p}=0.005)$.

\section{Gastric mucosal intestinal metaplasia among patients with Barrett's oesophagus}

We compared the prevalence of intestinal metaplasia among a subset of patients who had adequate gastric specimens available from both the antrum and the corpus ( 15 patients with Barrett's oesophagus and $24 \mathrm{H}$ pylori infected controls). None of the patients with Barrett's oesophagus had gastric intestinal metaplasia compared with 11 of the 24 patients with $H$ pylori gastritis $(\mathrm{p}=0.015)$. Nine cases from the 
control group with intestinal metaplasia were infected with CagA positive $H$ pylori. The site of intestinal metaplasia in the CagA positive patients was the antrum alone in five and the antrum and the corpus in four. Both CagA negative cases had antral intestinal metaplasia.

\section{DISCUSSION}

Colombia is typical of a developing country in that $H$ pylori infection is present in most adults, with most individuals being infected with a CagA positive strain. ${ }^{21-23}$ Gastric cancer is common in Colombia, ${ }^{30}{ }^{31}$ but the association between $H$ pylori (or a particular strain) and GERD/Barrett's oesophagus has not been adequately studied.

Our study examined the relation between $H$ pylori and long segment Barrett's oesophagus in Colombia. As we anticipated, $H$ pylori infection was present in most Colombians with long segment Barrett's oesophagus (32 of 51; 62.7\%), which is higher than the prevalence of $H$ pylori among patients with Barrett's oesophagus in the USA $(10-32.9 \%) .{ }^{10}{ }^{32-34}$ Our second hypothesis was that most $H$ pylori infected patients with Barrett's oesophagus would have CagA positive $H$ pylori infections, but this was disproved because CagA positive $H$ pylori was present in only approximately one third of those with active $H$ pylori infections. This frequency is higher than the prevalence reported among patients with Barrett's oesophagus in the USA (0-15.0\%), ${ }^{17-20}$ and much lower than the prevalence reported among the general population of $H$ pylori infected Colombians. ${ }^{22}{ }^{35}$ Our data are reminiscent of a study in Italy where CagA positive $H$ pylori infections were present in $42 \%$ of patients with Barrett's oesophagus compared with $80 \%$ of control infected patients $(p<0.03) .{ }^{36}$ In addition, the gastric mucosa of Italian patients with Barrett's oesophagus was typically non-atrophic: intestinal metaplasia was seen in $13 \%$ of the patients with Barrett's oesophagus compared with $30 \%$ of controls $(p<0.03){ }^{36}$

\section{"None of the patients with Barrett's oesophagus had intestinal metaplasia in gastric mucosal biopsies, whereas intestinal metaplasia was found in 11 of 24 controls with H pylori gastritis"}

In Colombia, as elsewhere, Barrett's oesophagus is primarily a disease of older men. ${ }^{37}{ }^{38}$ Interestingly, none of the Colombian women with long segment Barrett's oesophagus had CagA positive $H$ pylori infections. In addition, none of the patients with Barrett's oesophagus had intestinal metaplasia in gastric mucosal biopsies, whereas intestinal metaplasia was found in 11 of 24 controls with $H$ pylori gastritis. These data are consistent with the hypothesis that Barrett's oesophagus is related to the degree and severity of gastro-oesophageal reflux, reflected in the oesophageal acid load. ${ }^{13}$ Reduced acid secretion is associated with corpus gastritis, such that infection with CagA positive $H$ pylori

\section{Take home messages}

- We investigated the relation between Barrett's oesophagus and Helicobacter pylori infection in Colombia and found that although most patients were infected, CagA positive infections were unusual

- This supports the hypothesis that consistent corpus inflammation reduces acid secretion, which prevents Barrett's oesophagus among those with abnormal gastro-oesophageal reflux barriers would be expected to cause a more intense reduction than infection with CagA negative infections. ${ }^{13}{ }^{14}$ Colombians with long segment Barrett's oesophagus are somewhat unusual because they were more likely to be infected with CagA negative $H$ pylori and not to have intestinal metaplasia in the stomach.

Our study focused on the relation between the presence of $H$ pylori infection, particularly the cag pathogenicity island, and Barrett's oesophagus. There are several other host and $H$ pylori related factors that may also be important in reducing or enhancing the odds of developing a particular outcome other than the presence of the cag pathogenicity island. For example, genetic polymorphisms in proflammatory cytokine genes can result in an enhanced inflammatory response to any stimulus (such as periodontal disease), independent of whether $H$ pylori infection is present, ${ }^{39}$ and have been shown to be important in gastric ${ }^{40}$ and oesophageal carcinoma. ${ }^{41}$ Alterations in host factors, such as Lewis antigens, may also be important in relation to $H$ pylori infection ${ }^{42}$; however, polymorphisms in HLA antigens have not been shown to be important. ${ }^{43}$ Understanding the interactions between host, $H$ pylori, and environmental factors is difficult, because synergistic effects between environmental, host (for example, genetic polymorphisms), and $H$ pylori virulence factors need to be taken into account. ${ }^{39445}$ Authors are only allowed one affiliation each.

\section{ACKNOWLEDGEMENTS}

This material is based upon work supported in part by the Office of Research and Development Medical Research Service Department of Veterans Affairs and by Public Health Service grant DK56338, which funds the Texas Gulf Coast Digestive Diseases Center. The authors thank Professor M Asaka, Hokkaido University Graduate School of Medicine for support and T Kudo, Baylor College of Medicine, for many helpful comments.

\section{Authors' affiliations}

M Kudo, H M T El-Zimaity, Z Z Nurgalieva, J Wu, D Y Graham, Michael E DeBakey Veterans Affairs Medical Center and Baylor College of Medicine, Houston, TX 77030, USA

M Kudo, Hokkaido University, Graduate School of Medicine,

Department of Internal Medicine, Gastroenterology and Haematology Section, Kita 15 jo, Nishi 7 Chome, Kita-Ku, Sapporo Hokkaido, 0608638, Japan

O Gutierrez, H Cardona, National University, Department of Internal Medicine, Gastroenterology Unit, Bogotá, Colombia

\section{REFERENCES}

1 Falk GW. Reflux disease and Barrett's esophagus. Endoscopy 1999;31:9-16.

2 Cameron AJ. Epidemiology of Barrett's esophagus and adenocarcinoma. Dis Esophagus 2002;15:106-8.

3 El-Serag HB. The epidemic of esophageal adenocarcinoma. Gastroenterol Clin North Am 2002;31:421-40, viii.

4 El-Serag HB, Mason AC, Petersen N, et al. Epidemiological differences between adenocarcinoma of the oesophagus and adenocarcinoma of the gastric cardia in the USA. Gut 2002;50:368-72.

5 Genta RM, Huberman RM, Graham DY. The gastric cardia in Helicobacter pylori infection. Hum Pathol 1994;25:915-19.

6 Graham DY. Helicobacter pylori is not and never was "protective" against anything, including GERD. Dig Dis Sci 2003;48:629-30.

7 Graham DY. Campylobacter pylori and Barrett's esophagus. Mayo Clin Proc 1988;63:1258-60.

8 Blaser MJ, Perez-Perez Gl, Lindenbaum J, et al. Association of infection due to Helicobacter pylori with specific upper gastrointestinal pathology. Rev Infect Dis 1991;13(suppl 8):S704-8

9 Csendes A, Smok G, Cerda G, et al. Prevalence of Helicobacter pylori infection in 190 control subjects and in 236 patients with gastroesophageal reflux, erosive esophagitis or Barrett's esophagus. Dis Esophagus 1997; 10:38-42.

10 Henihan RD, Stuart RC, Nolan N, et al. Barrett's esophagus and the presence of Helicobacter pylori. Am J Gastroenterol 1998;93:542-6.

11 Kogan Z, Corti R, Cabane A, et al. Helicobacter pylori in Barrett esophagus. Acta Gastroenterol Latinoam 1992;22:215-19.

12 Laheij RJ, van Rossum LG, de Boer WA, et al. Corpus gastritis in patients with endoscopic diagnosis of reflux oesophagitis and Barrett's oesophagus. Aliment Pharmacol Ther 2002;16:887-91. 
13 Graham DY. The changing epidemiology of GERD: geography and Helicobacter pylori. Am J Gastroenterol 2003;98: 1462-70.

14 Graham DY, Yamaoka Y. H. pylori and cagA: relationships with gastric cancer, duodenal ulcer, and reflux esophagitis and its complications, Helicobacter 1998:3:145-51.

15 Graham DY, Malaty HM, Evans DG, et al. Epidemiology of Helicobacter pylori in an asymptomatic population in the United States. Effect of age, race and socioeconomic status. Gastroenterology 1991;100:1495-501.

16 Shiotani A, Graham DY. Pathogenesis and therapy of gastric and duodenal ulcer disease. Med Clin North Am 2002;86:1447-66.

17 Quddus MR, Henley JD, Sulaiman RA, et al. Helicobacter pylori infection and adenocarcinoma arising in Barrett's esophagus. Hum Pathol 1997:28:1007-9.

18 Vaezi MF, Falk GW, Peek RM, et al. CagA-positive strains of Helicobacter pylori may protect against Barrett's esophagus. Am J Gastroenterol 2000:95:2206-11.

19 Loffeld RJ, Werdmuller BF, Kuster JG, et al. Colonization with cagA-positive Helicobacter pylori strains inversely associated with reflux esophagitis and Barrett's esophagus. Digestion 2000;62:95-9.

20 Vicari JJ. The seroprevalence of cagA-positive Helicobacter pylori strains in the spectrum of gastroesophageal reflux disease. Gastroenterology 1998;115:50-7.

21 Brever T, Malaty HM, Graham DY. The epidemiology of $H$. pylori-associated gastroduodenal diseases. In, Ernst P, Michetti P, Smith PD, eds. The immunobiology of $\mathrm{H}$. pylori from pathogenesis to prevention. Philadelphia, Lippincott-Raven, 1997: 1-14.

22 Yamaoka Y, Kodama T, Gutierrez O, et al. Relationship between Helicobacter pylori iceA, cagA, and vacA status and clinical outcome: studies in four different countries. J Clin Microbiol 1999;37:2274-9.

23 Yamaoka Y, Osato MS, Sepulveda AR, et al. Molecular epidemiology of Helicobacter pylori: separation of $\mathrm{H}$. pylori from East Asian and non-Asian countries. Epidemiol Infect 2000;124:91-6.

24 Nogueira C, Figueiredo C, Carneiro F, et al. Helicobacter pylori genotypes may determine gastric histopathology. Am J Pathol 2001;158:647-54.

25 Genta RM, Robason GO, Graham DY. Simultaneous visualization of Helicobacter pylori and gastric morphology: a new stain. Hum Pathol 1994;25:221-6.

26 El-Zimaity HM, Ota H, Scott S, et al. A new triple stain for Helicobacter pylori suitable for the autostainer: carbol fuchsin/Alcian blue/hematoxylin-eosin. Arch Pathol Lab Med 1998;122:732-6.

27 El-Zimaity HM. Modified triple stain (carbol Fuchsin/Alcian blue/ hematoxylin-eosin) for the identification of Helicobacter pylori. Arch Pathol Lab Med 2000; 124:1417.

28 El-Zimaity HM, Graham DY, Al-Assi MT, et al. Interobserver variation in the histopathological assessment of Helicobacter pylori gastritis. Hum Pathol 1996:27:35-41.
29 Park CY, Kwak M, Gutierrez O, et al. Comparison of genotyping Helicobacter pylori directly from biopsy specimens and genotyping from bacterial cultures. $J$ Clin Microbiol 2003;41:3336-8.

30 Correa P, Fox J, Fontham E, et al. Helicobacter pylori and gastric carcinoma. Serum antibody prevalence in populations with contrasting cancer risks. Cancer 1990;66:2569-74.

31 Cuello C, Correa P, Haenszel W, et al. Gastric cancer in Colombia. I. Cancer risk and suspect environmental agents. J Natl Cancer Inst 1976;57:1015-20.

32 Peek RM Jr, Vaezi MF, Falk GW, et al. Role of Helicobacter pylori cagA+ strains and specific host immune responses on the development of premalignant and malignant lesions in the gastric cardia. Int $J$ Cancer 1999;82:520-4.

33 Weston AP, Badr AS, Topalovski M, et al. Prospective evaluation of the prevalence of gastric Helicobacter pylori infection in patients with GERD, Barrett's esophagus, Barrett's dysplasia, and Barrett's adenocarcinoma. Am J Gastroenterol 2000;95:387-94.

34 Clark GW. Effect of Helicobacter pylori infection in Barrett's esophagus and the genesis of esophageal adenocarcinoma. World J Surg 2003;27:994-8.

35 Goodman KJ, Correa P, Tengana Aux HJ, et al. Helicobacter pylori infection in the Colombian Andes: a population-based study of transmission pathways. Am J Epidemiol 1996;144:290-9.

36 Rugge M, Russo V, Busatto $G$, et al. The phenotype of gastric mucosa coexisting with Barrett's oesophagus. J Clin Pathol 2001;54:456-60.

37 Cameron AJ, Lomboy CT. Barrett's esophagus: age, prevalence, and extent of columnar epithelium. Gastroenterology 1992;103:1241-5.

38 Cameron AJ. Epidemiology of columnar-lined esophagus and adenocarcinoma. Gastroenterol Clin North Am 1997;26:487-94.

39 Dore MP, Graham DY. Ulcers and gastritis. Endoscopy 2004;36:42-7.

40 El-Omar EM, Carrington M, Chow WH, et al. The role of interleukin-1 polymorphisms in the pathogenesis of gastric cancer. Nature 2001;412:99.

41 el-Omar EM, Rabkin CS, Gammon MD, et al. Increased risk of noncardia gastric cancer associated with proinflammatory cytokine gene polymorphisms. Gastroenterology 2003;124:1193-201.

42 Ikehara Y, Nishihara S, Yasutomi H, et al. Polymorphisms of two fucosyltransferase genes (Lewis and Secretor genes involving type I Lewis antigens) are associated with the presence of anti-Helicobacter pylori lgG antibody. Cancer Epidemiol Biomarkers Prev 2001;10:971-7.

43 Kunstmann E, Hardt C, Crabtree JE, et al. Helicobacter pylori infection: CagA-specific antibodies are associated with clinical outcome, but not with HLA-class II polymorphisms of the host. Int J Med Microbiol 2003;292:537-40

44 Rad R, Prinz C, Neu B, et al. Synergistic effect of Helicobacter pylori virulence factors and interleukin-1 polymorphisms for the development of severe histological changes in the gastric mucosa. J Infect Dis 2003;188:272-81.

$45 \operatorname{Rad} \mathbf{R}$, Dossumbekova A, Neu B, et al. Cytokine gene polymorphisms influence mucosal cytokine expression, gastric inflammation, and host specific colonisation during Helicobacter pylori infection. Gut 2004;53:1082-9. 